University of Wollongong

Research Online

Faculty of Engineering and Information

Faculty of Engineering and Information

Sciences - Papers: Part A

Sciences

$1-1-2013$

\title{
Neutron generator burst timing measured using a pulse shape discrimination plastic scintillator with silicon photomultiplier readout
}

R M. Preston

University of Wollongong, rmp987@uowmail.edu.au

$J$ Eberhardt

CSIRO

J R. Tickner

CSIRO, james.tickner@csiro.au

Follow this and additional works at: https://ro.uow.edu.au/eispapers

Part of the Engineering Commons, and the Science and Technology Studies Commons

Research Online is the open access institutional repository for the University of Wollongong. For further information contact the UOW Library: research-pubs@uow.edu.au 


\title{
Neutron generator burst timing measured using a pulse shape discrimination plastic scintillator with silicon photomultiplier readout
}

\author{
Abstract \\ An EJ-299-34 plastic scintillator with silicon photomultiplier (SiPM) readout was used to measure the fast \\ neutron output of a pulsed Thermo-Fisher A-325 Deuterium-Tritium sealed tube neutron generator \\ (STNG). The SiPM signals were handled by a prototype digital pulse processing system, based on a free- \\ running analogue to digital converter feeding a digital signal processor (DSP). Pulse shape discrimination \\ was used to distinguish between detected fast-neutrons and gammas. Pulse detection, timing, energy \\ and shape were all processed by the DSP in real-time.
}

\section{Keywords}

measured, timing, discrimination, burst, photomultiplier, generator, readout, scintillator, pulse, shape, plastic, silicon, neutron

Disciplines

Engineering | Science and Technology Studies

\section{Publication Details}

Preston, R. M., Eberhardt, J. \& Tickner, J. R. (2013). Neutron generator burst timing measured using a pulse shape discrimination plastic scintillator with silicon photomultiplier readout. Journal of Instrumentation, 8 P12005-1-P125001-12. 


\title{
Neutron Generator Burst Timing measured using a Pulse Shape Discrimination Plastic Scintillator with Silicon Photomultiplier Readout
}

\author{
Rhys M. Preston, ${ }^{a, b^{*}}$ John E. Eberhardt ${ }^{a}$ and James R. Tickner ${ }^{a}$ \\ ${ }^{a}$ CSIRO Process Science and Engineering / CSIRO Minerals Down Under Research Flagship, \\ New Illawarra Rd, Lucas Heights, Australia \\ ${ }^{b}$ Centre for Medical Radiation Physics, University of Wollongong \\ Northfields Ave, Wollongong, Australia \\ E-mail: rhys.prestonecsiro.au
}

ABSTRACT: An EJ-299-34 plastic scintillator with silicon photomultiplier (SiPM) readout was used to measure the fast neutron output of a pulsed Thermo-Fisher A-325 Deuterium-Tritium sealed tube neutron generator (STNG). The SiPM signals were handled by a prototype digital pulse processing system, based on a free-running analogue to digital converter feeding a digital signal processor (DSP). Pulse shape discrimination was used to distinguish between detected fast-neutrons and gammas. Pulse detection, timing, energy and shape were all processed by the DSP in real-time.

The time-dependency of the neutron output of the STNG was measured for various pulsing schemes. The switch-on characteristics of the tube strongly depended on the operating settings, with the delay between pulse turn-on and the production of neutrons ranging between $13 \mu$ s to $74 \mu$ s for the tested pulse rates and duty cycles. This work will facilitate the optimization and modeling of apparatus that use the neutron generator's pulsing abilities.

KEYWORDS: Instrumentation for neutron sources; Digital signal processing (DSP); Photon detectors for UV, visible and IR photons (solid-state) (PIN diodes, APDs, Si-PMTs, G-APDs, CCDs, EBCCDs, EMCCDs etc).

${ }^{*}$ Corresponding author. 


\section{Contents}

1. Introduction 1

2. Fast Neutron Detector 2

2.1 Detector 3

2.2 Digital Pulse Processor 3

2.2.1 Pulse Detection 4

2.2.2 Timing 4

2.2.3 Weighted Integration 5

2.2.4 System Dead Time $\quad 6$

2.3 Host 6

3. Measurement 6

$\begin{array}{ll}\text { 4. Results } & 7\end{array}$

5. Conclusions $\quad 10$

\section{Introduction}

The sealed tube neutron generator (STNG) is a class of accelerator-based fast-neutron source used in scientific and applied research. Some STNG applications include the detection of contraband and explosives [1,2], geophysical logging [3,4], and fast-neutron/gamma radiography $[5,6]$.

STNGs commonly use the Deuterium-Deuterium (DD) or Deuterium-Tritium (DT) fusion reactions. Deuterons from an ion source are accelerated towards a tritiated or deuterated target. Both reactions are exothermic, so $\mathrm{MeV}$ energy neutrons may be produced using moderate accelerating voltages suitable for use in a small tube, typically in the 50-150 kV range [7,8]. DD tubes produce $\sim 2.45 \mathrm{MeV}$ neutrons while DT tubes produce $\sim 14.1 \mathrm{MeV}$ neutrons.

A number of techniques make use of the STNGs ability to modulate the output of neutrons. Pulsed Fast/Thermal Neutron Analysis (PFTA) allows the collection of gamma spectra corresponding to inelastic scattering (n,n'y) of fast neutrons during the neutron burst and gamma spectra due to $(n, \gamma)$ capture of moderated neutrons after each burst [9]. Other techniques, such as pulsed neutron logging [10,11] and Differential Die Away Analysis (DDAA) [12], rely on measuring the population of epithermal or thermal neutrons in the time following each neutron burst. 
Neutron pulsing is achieved by switching the STNG ion source on and off. However the time profile of the ion generation, and hence neutron output, may not exactly follow the switching applied to the source. In particular, there can be a significant delay between the switch-on of the ion source and the steady production of ions, depending on the type of ion source used [13]. Consequently, the measurement of the neutron output over the duration of the burst cycle is necessary to effectively model and optimize systems that utilize a pulsed STNG.

The following paper describes the application of a recently developed fast neutron detector to the measurement of the burst characteristics of a Thermo-Fisher A-325 with A-3062 DT tube. The A-3062 is a passively cooled tube, with a specified maximum output of approximately $10^{8}$ neutrons/s into $4 \pi$ steradian. The stability of the STNG output during DC operation was previously measured with $\sim 10 \mathrm{~ms}$ resolution using an EJ-204 plastic scintillator detector [14]. The A-325 control unit includes a signal generator for gating the HV supply to the ion source with a specified $10 \mu \mathrm{s}$ minimum pulse width. Like most STNGs intended for industrial applications $[7,15]$, the generator utilizes a cold-cathode Penning ion source.

\section{Fast Neutron Detector}

To characterise the STNG in burst mode, detection of the generated fast neutrons was necessary. Fast neutrons may be detected using an organic scintillator to detect the recoil protons produced by fast neutron scattering with hydrogen nuclei $[16,17]$. Organic scintillators are also sensitive to gamma radiation, so gamma-ray induced scintillations may present a background that limits the effective measurement of fast-neutron flux. The gamma radiation may be natural background or may result from neutron interactions with the surrounding environment.

With some types of organic scintillator, the time profile of the scintillation photon emission depends on the ionization densities produced by the ionizing particle [16]. The proportion of faster ( $\sim 5 \mathrm{~ns}$ and $\sim 30 \mathrm{~ns})$ to slower (>100 ns) components of the scintillation decay is reduced with higher ionization densities. Because neutron-recoiled nuclei are more densely ionizing than gamma-ray induced energetic electrons, the pulse shapes may be analyzed and used to separately measure the fast neutron and gamma components of a mixed radiation field. This technique is known as Pulse Shape Discrimination (PSD). Organic scintillators that exhibit useful PSD include some liquid scintillators, some organic crystals and some recently developed plastics $[18,19]$.

Traditionally pulse shape discriminating fast-neutron detectors use a photomultiplier tube (PMT) for optical readout. However, it has been demonstrated that a Silicon Photomultiplier (SiPM) can be a viable alternative to the PMT for measuring PSD in small organic [20,21] and inorganic scintillators $[22,23]$. The SiPM is a type of solid state photo-detector with intrinsic gains and photon detection efficiencies similar to PMTs [24,25]. Using an SiPM significantly reduces the potential cost and size of these detectors.

We are developing a fast-neutron detection system appropriate for handheld use. This uses a PSD plastic scintillator with SiPM readout. Digital pulse processing is implemented using a free-running analogue to digital converter (ADC) and digital signal processor (DSP). As the prototype supported time-resolved measurements of fast neutrons with gamma discrimination, it was well-suited to the measurement of the neutron burst profile. The following describes the prototype system used to characterize the STNG. 


\subsection{Detector}

The detector was based around a $1 \times 1 \times 5 \mathrm{~cm}$ EJ-299-34 plastic scintillator from Eljen Technology. The EJ-299-34 was a developmental-prototype based on the PSD plastic that was invented at Lawrence Livermore National Laboratory [18] and is being commercialized by Eljen Technology [19]. A SensL FB60035 $6 \times 6 \mathrm{~mm}$ SiPM was used for the optical readout. This was coupled to one square end of the scintillator using an EJ-560 optical interface pad. The scintillator was wrapped with 3M Vikuiti Enhanced Specular Reflector. One-wrap Teflon tape was used to cover small gaps in the reflector. The detector is shown in Figure 1. A small printed circuit board (PCB) was used to interface with the SiPM surface-mount package and provide a bias decoupling network. The scintillator side of the PCB was painted with titanium dioxide white paint to form part of the reflector. The detector was operated in a small and light tight tinplated steel enclosure with BNC bulkhead sockets used to connect the SiPM bias and anode output signal.

\subsection{Digital Pulse Processor}

A digital pulse processor (DPP) is being developed at CSIRO for handling SiPM pulses. This DPP measures the energy, pulse shape and time of arrival of each detected pulse. The DPP hardware was based around an Analog Devices Blackfin BF-533 digital signal processor (DSP). The initial prototype used in this work, shown in Figure 1, was put together using an ADSP-BF533-EZkit-Lite DSP development kit and an AD6645SQZ ADC evaluation board. The block diagram of the DPP is shown in Figure 2.
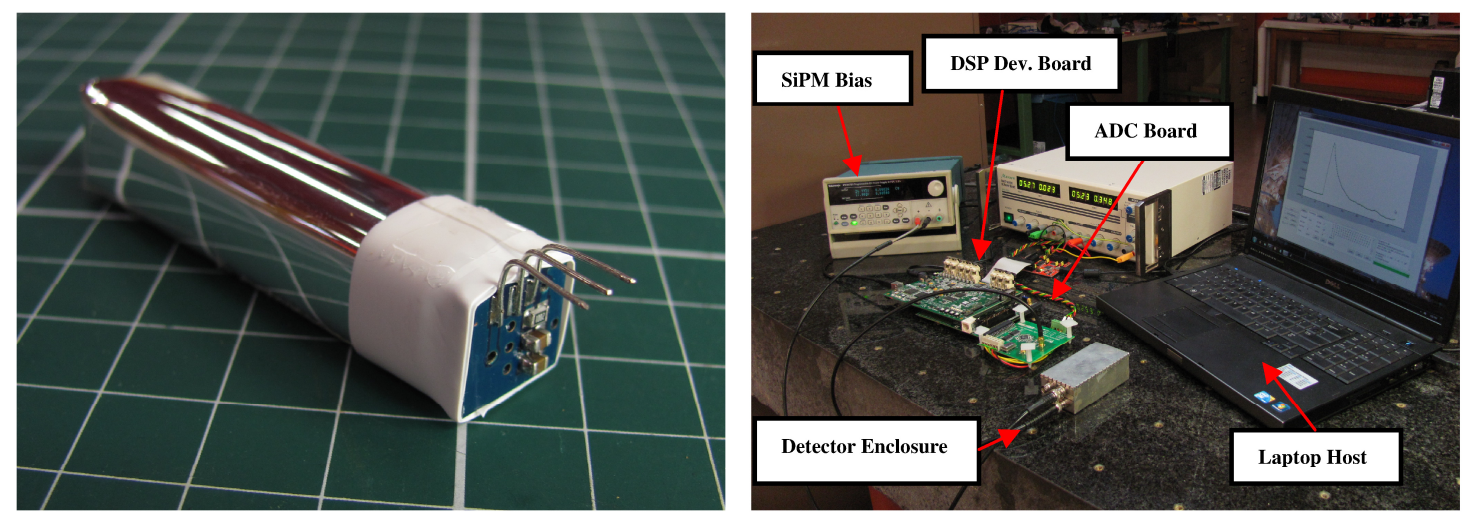

Figure 1: EJ-299-34 scintillator detector with FB60035 SiPM readout (left) and the prototype detection system used for the measurements (right). For scale, the small grid squares in the left photograph measure $1 \times 1 \mathrm{~cm}$.

The SiPM signal was sampled by an AD6645 14 bit ADC clocked at $50 \mathrm{MSample} / \mathrm{s}$ (MSps). An AD8138 differential amplifier was used to convert the single ended SiPM signals into differential ones appropriate for the ADC input. The amplifier gain and offset were adjusted to make full use of the ADC input range. A feedback capacitor provided a simple low-pass Nyquist filter with cut-off at approximately $20 \mathrm{MHz}$.

The parallel outputs of the ADC interfaced with the Blackfin using the Parallel Peripheral Interface (PPI). The Blackfin Direct Memory Access (DMA) engine was set-up in auto-buffer mode, where the ADC samples were moved to an 8192 sample circular buffer in level 1 (L1) 
memory. The buffer was partitioned into four sub-buffers, which were filled and subsequently processed in a block-processing setup.

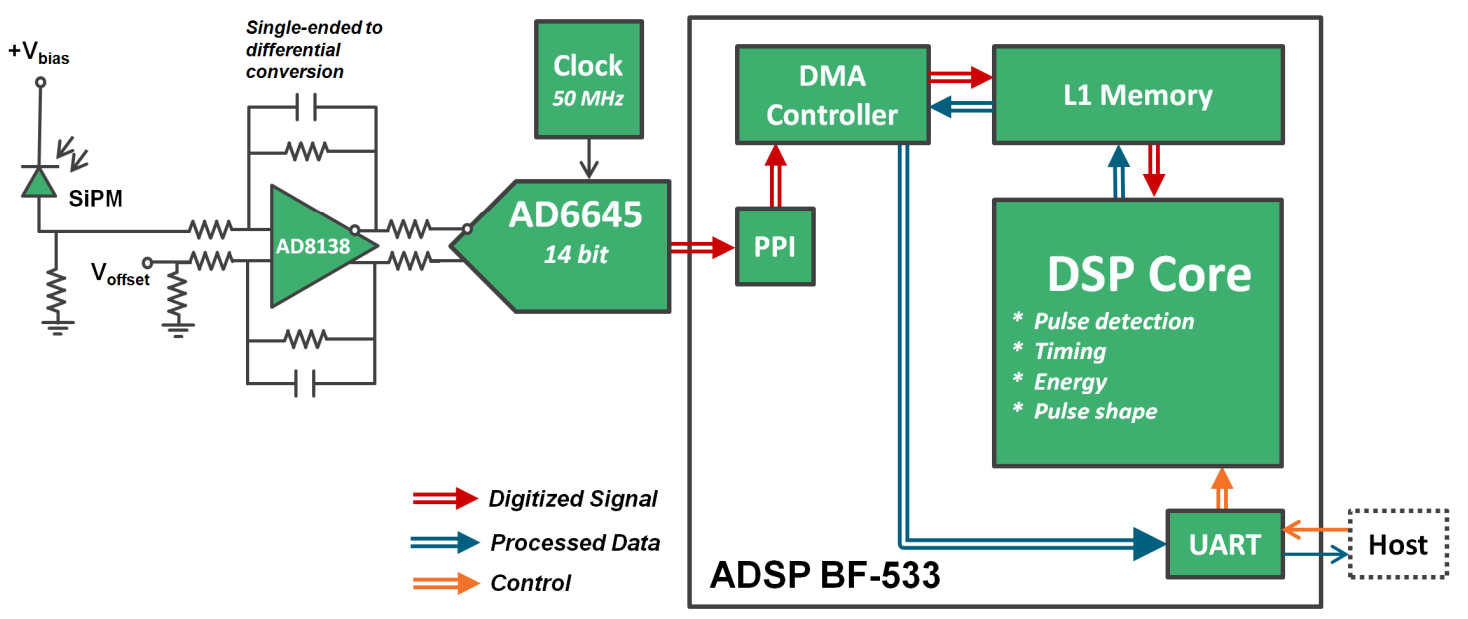

Figure 2: Simplified block diagram of the DPP hardware based around a Blackfin DSP

The core performed all pulse processing functions; pulse detection, timing, energy and shape measurement. As the core operated at $600 \mathrm{MHz}$ and the sample rate was $50 \mathrm{MSps}$ the processing must take, on average, less than 12 clock cycles to process each sample. The detection and measurement algorithms described below were coded in Blackfin assembly language to utilize the DSP capabilities such as hardware looping, parallel instructions, static branch prediction and hardware circular addressing. The sub-routines were aware of the circular buffer, so pulses falling across the end of the buffer (wrapping around to the beginning) were processed as normal. The less performance-critical parts of the program were coded in C. Using this approach the DSP required less than 6 cycles per sample for processing signals with incident neutron and gamma-ray count rates up to $100 \mathrm{kcps}$, satisfying the real-time constraint.

\subsubsection{Pulse Detection}

SiPMs produce thermally triggered pulses, identical to those triggered by detected photons, at rates of around 0.1-1 Mcps per $\mathrm{mm}^{2}$ of active area [26]. These so-called dark pulses produce significant baseline disturbance in the output signal and, as a result, a level discriminator was not an ideal method for detecting the presence of a scintillation pulse. Instead, the pulse detection sub-routine searched for the rapid rise in signal associated with the leading edge of each scintillation pulse. The forward finite difference $\Delta v$ of the digitised signal $v$ was calculated:

$$
\Delta v_{i}=v_{i+l}-v_{i}
$$

where the window length $l$ was user-adjustable. Pulses were identified where $\Delta \boldsymbol{v}$ exceeded a user-defined threshold. Figure 3 shows the $\Delta v$ calculated for SiPM signals digitized by the DPP.

\subsubsection{Timing}

Once a pulse was detected, the trace following the trigger was scanned for the local minimum (base) and maximum (peak). The pulse height was the difference in height between these points. The time of arrival was estimated at the sample where the leading edge exceeded $50 \%$ of the pulse height above the base. 


\subsubsection{Weighted Integration}

The energy $E$ and pulse shape $S$ of each pulse were calculated using weighted integrations:

$$
S=\sum_{i=1}^{n} p_{i} \cdot w_{i},
$$

where the $n$ samples of each detected and aligned pulse $\boldsymbol{p}$ were multiplied by a corresponding coefficient in the weighting vector $\boldsymbol{w}$ and accumulated to give the measured result. This is a digital implementation of a linear filter [27]. A weighting vector for each measurement was uploaded to the DSP prior to acquisition. The weighting vectors were balanced (the sum of the coefficients were zero) to enforce a correction for any DC offset in the trace. For energy measurement, each scintillation was integrated using a weighting vector with rectangular coefficients, with a $2 \mu \mathrm{s}$ window over the pulse and a $500 \mathrm{~ns}$ baseline window in the time preceding the pulse.

For PSD, $w$ was to be set such that $S$ characterized the scintillation time profile. Because $S$ was also proportional to the amplitude of the pulse, $S$ was normalized using the measured energy $E$. There are a number of methods for selecting $\boldsymbol{w}$ for the measurement of $S$. Digital Charge Comparison (DCC) is a commonly used technique that may be implemented as a weighted integration using rectangular coefficients with an integration that is shorter or delayed with respect to the energy integration [28]. If the digitized pulse from the detector accurately represents the time profile of the scintillation, as is the case with many PMT-based detectors, the optimum weights may be calculated analytically from the average gamma and neutron pulse shapes $[27,29]$.
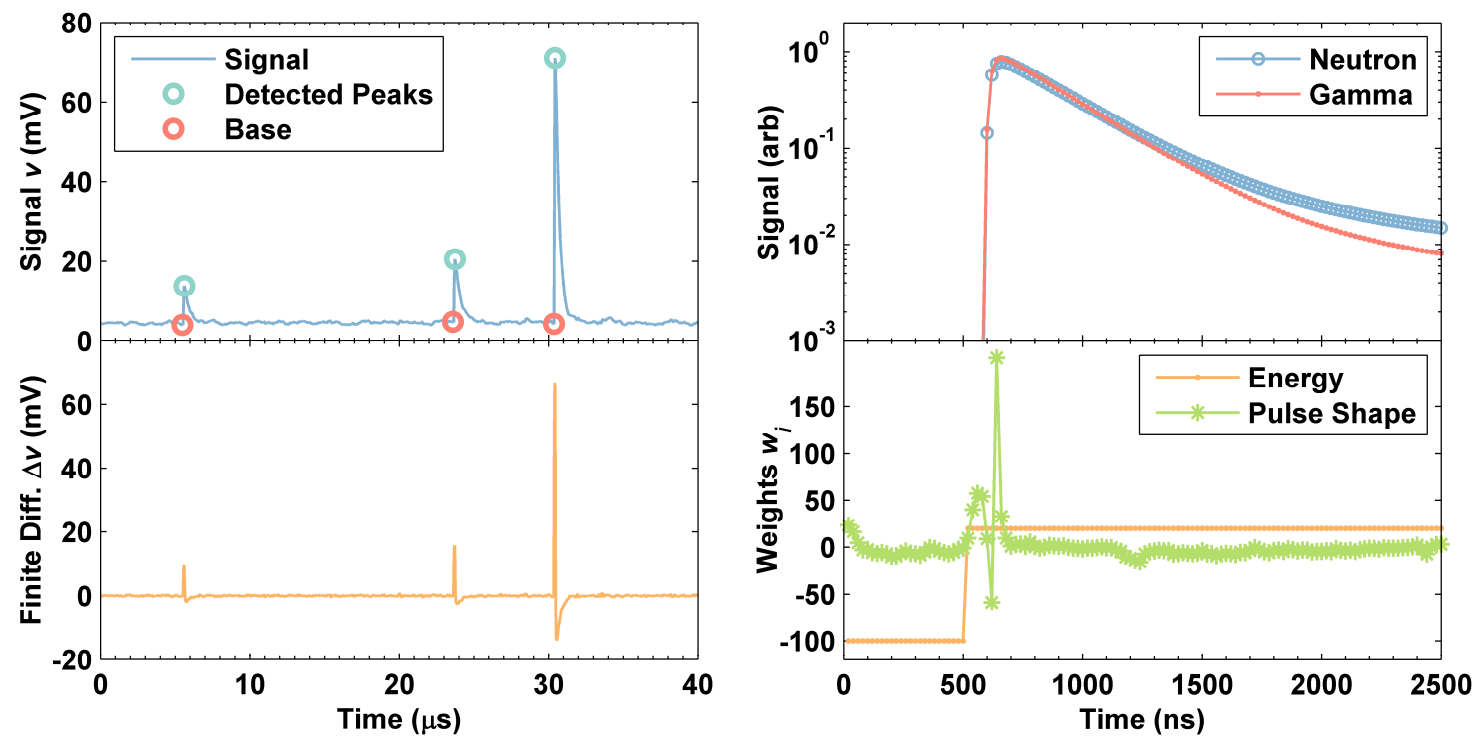

Figure 3: Detected pulses in a 2000 sample sub-buffer and the finite differenced used for detection (left). Average profiles of neutron and gamma pulses detected and aligned by the DPP system and the weighting vectors used to extract energy and pulse shape information (right). With the average profiles normalised according to energy, the neutron profile exhibit a greater proportion of signal in the tail of the pulse.

When using an SiPM-based detector, the characteristic SiPM pulse shape and noise complicates the analytical approach. Instead, a numerical optimization was used to estimate the 
optimum $\boldsymbol{w}$. This was performed using a real-encoded genetic algorithm (GA). GA is a type of search heuristic inspired by evolutionary 'survival of the fittest' models in nature and is based on combining and mutating generations of provisional solutions [30]. The GA searched for the weights that gave the optimum PSD for a bank of collected and identified neutron and gamma training pulses [21]. A set of neutron and gamma pulses were collected using the DPP pulsecapture function. These pulses were then identified offline using DCC and fed to the GA to generate an optimized weighting vector. The optimized weighting vector, displayed in Figure 3, was then uploaded to the DSP for the online measurements.

\subsubsection{System Dead Time}

After a scintillation event was detected and processed, the pulse detection sub-routine resumed at a fixed number of samples following the measured pulse time. This hold-off time was set according to the length of the weighting vectors. For the following measurements the weighting vectors were 125 samples long, fixing the dead time $\tau_{\text {dead }}$ at $2.5 \mu \mathrm{s}$. The system approximates the non-paralysable dead time model [31], where the true count rate $n$ may be calculated from the observed rate $\mathrm{m}$ :

$$
n=\frac{m}{1-m \tau_{\text {dead }}} .
$$

\subsection{Host}

The DSP communicated with a host laptop computer using a built-in universal asynchronous receiver/transmitter (UART) interfaced to a FT2232 UART to USB 2.0 adapter. A graphical user interface for operating the DPP was written in python, using matplotlib [32] for plotting the collected data and pySerial [33] for the communications.

\section{Measurement}

The A-3062 DT neutron tube was situated in a shielding cave, made up of borated paraffin wax bricks. The detector was positioned with one square end facing the DT tube at a distance of $20 \mathrm{~cm}$ from the emission point. The SiPM was biased at $27 \mathrm{~V}$ (approximately $2.5 \mathrm{~V}$ above the FB60035 breakdown voltage) and the anode signal was connected to the DPP input via an RG58AU coaxial cable. A $100 \mathrm{~ns}$ rectangular pulse, pulsed at $10 \mathrm{~Hz}$ and synchronised with the neutron generator was used to provide a reference to the start of the pulsing cycle. This was also connected to the DPP input, parallel to the SiPM signal, via a $1 \mathrm{k} \Omega$ resistor. The pulse was distinctly different from the neutron and gamma pulses using the pulse shape parameter, $S$, so could be identified in post processing.

The A-325 was operated in a number of pulsing schemes, at 1,2 and $5 \mathrm{kHz}$ pulse rates and $10 \%, 20 \%$, and $50 \%$ duty cycles. The accelerator high voltage was fixed at $45 \mathrm{KV}$. The beam current, as indicated by the control unit, was adjusted before each measurement to be approximately $40 \pm 2 \mu \mathrm{A}$.

The digital pulse processor continuously acquired and processed $660 \mathrm{~s}$ of signals per run, dumping the list mode data to the host laptop. The detector was also used to measure a Na-22 source. The $341 \mathrm{keV}$ and $1057 \mathrm{keV}$ Compton edges in the Na-22 gamma spectrum, arising from the $511 \mathrm{keV}$ and $1275 \mathrm{keV}$ gamma-rays emitted by the source, were used to calibrate the energy scale in units of electron-equivalent $\mathrm{eV}\left(\mathrm{eV}_{\mathrm{ee}}\right)$. 


\section{Results}

The list mode data consisted of the measured scintillation energy and pulse shape with a timestamp corresponding to the time of arrival relative to the start of each 11 minute acquisition. To represent the profile of a single neutron burst, the pulse times were folded back to a single cycle by taking the modulus of the time with the pulsing period. Due to the slightly different crystal clocking of the pulse generator and the digitiser, the cycle period had to be adjusted by up to $\pm 1 \%$ from nominal for the folded times to align correctly. Furthermore, the relative clock rates drifted over the 11 minute measurements so the $10 \mathrm{~Hz}$ synchronised pulses were used to fine tune the times to correct for this shift.

The pulse shape and energies measured during and between the neutron bursts are shown in Figure 4. The in-burst 2D histogram has two main distributions, corresponding to protons recoiled by fast neutrons and electrons energized by gamma-rays. Counts between the two distributions are due to protons escaping the scintillator, as previously observed by another group using a similarly small-volume liquid scintillator [34]. The effect was more prevalent with the detector realigned such that a long side faced the neutron generator. Outside the STNG burst, only the gamma events are seen, occurring at a lower rate. The neutron and gamma-ray scintillations were separated by making cuts according to the pulse shape; example separated energy spectra are plotted in Figure 5. The endpoint of the neutron spectrum corresponds to the full deposition of $14 \mathrm{MeV}$ in the scintillator. The endpoint lies at $\sim 6 \mathrm{MeV}_{\mathrm{ee}}$ owing to the nonlinear response of the plastic scintillator to protons [35,36] and soft-saturation caused by the limited dynamic range of the SiPM [37].
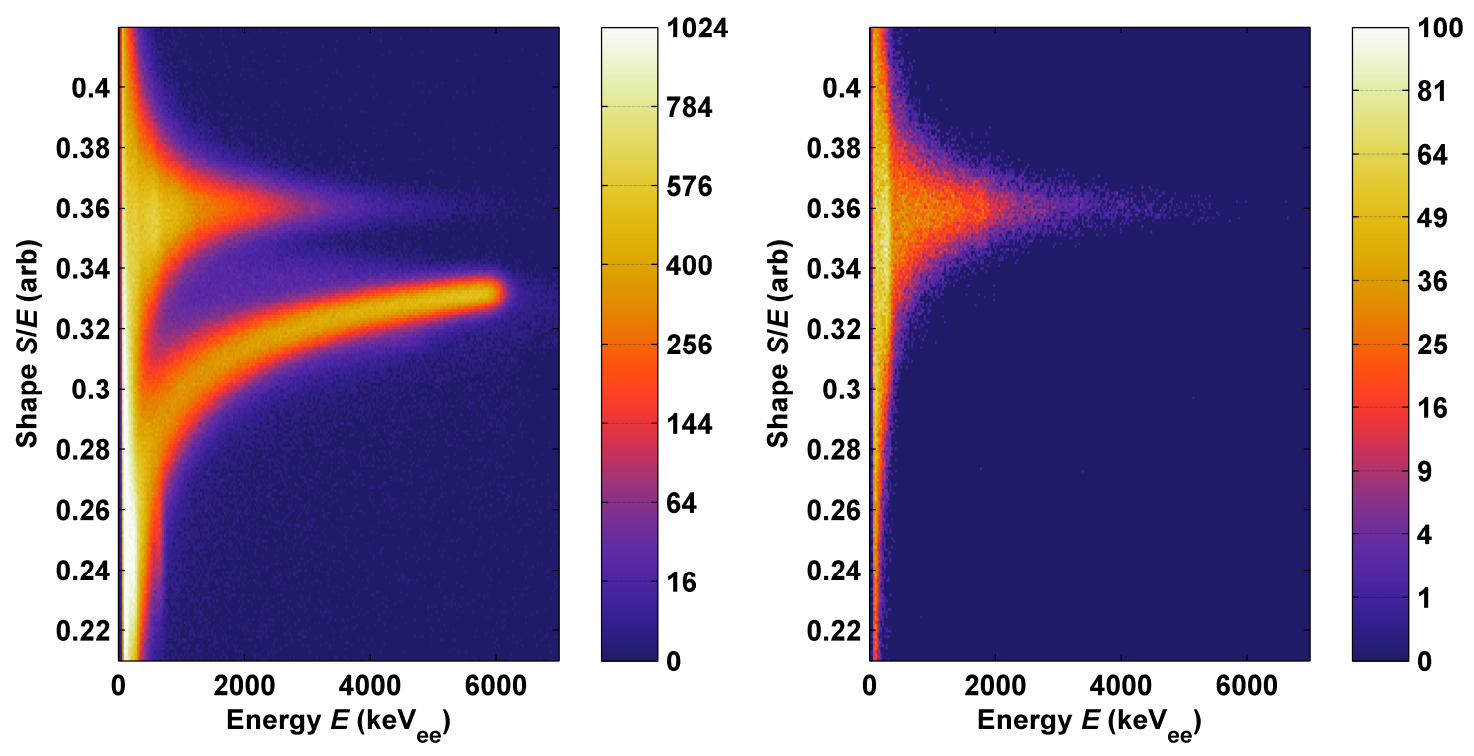

Figure 4: 2D Histogram of the PSD and Energy Values measured during (left) and between (right) neutron bursts. The colour scales represents measured counts. The lower distribution corresponds to the $14 \mathrm{MeV}$ fast neutron interactions while the upper population corresponds to gammas.

Figure 6 shows the neutron and gamma detections over the course of $2 \mathrm{kHz} 20 \%$ duty cycle burst period. Most of the gamma-ray counts follow the neutron output. These are gammarays produced in ( $\left.n, n^{\prime} y\right)$ inelastic scattering of fast neutrons. After neutron production is 
switched off, the remaining gamma-ray events show an exponential decay on top of an approximately constant background. The exponential decay exhibited a $124 \mu \mathrm{s}$ time constant, corresponding to the mean life time of moderated thermal neutrons in the shielding cave. Thermal neutron mean lifetimes typically range from $70 \mu$ s to $500 \mu$ s [10], depending on the surrounding materials. The constant offset is attributed to the decays of neutron-induced activation products.

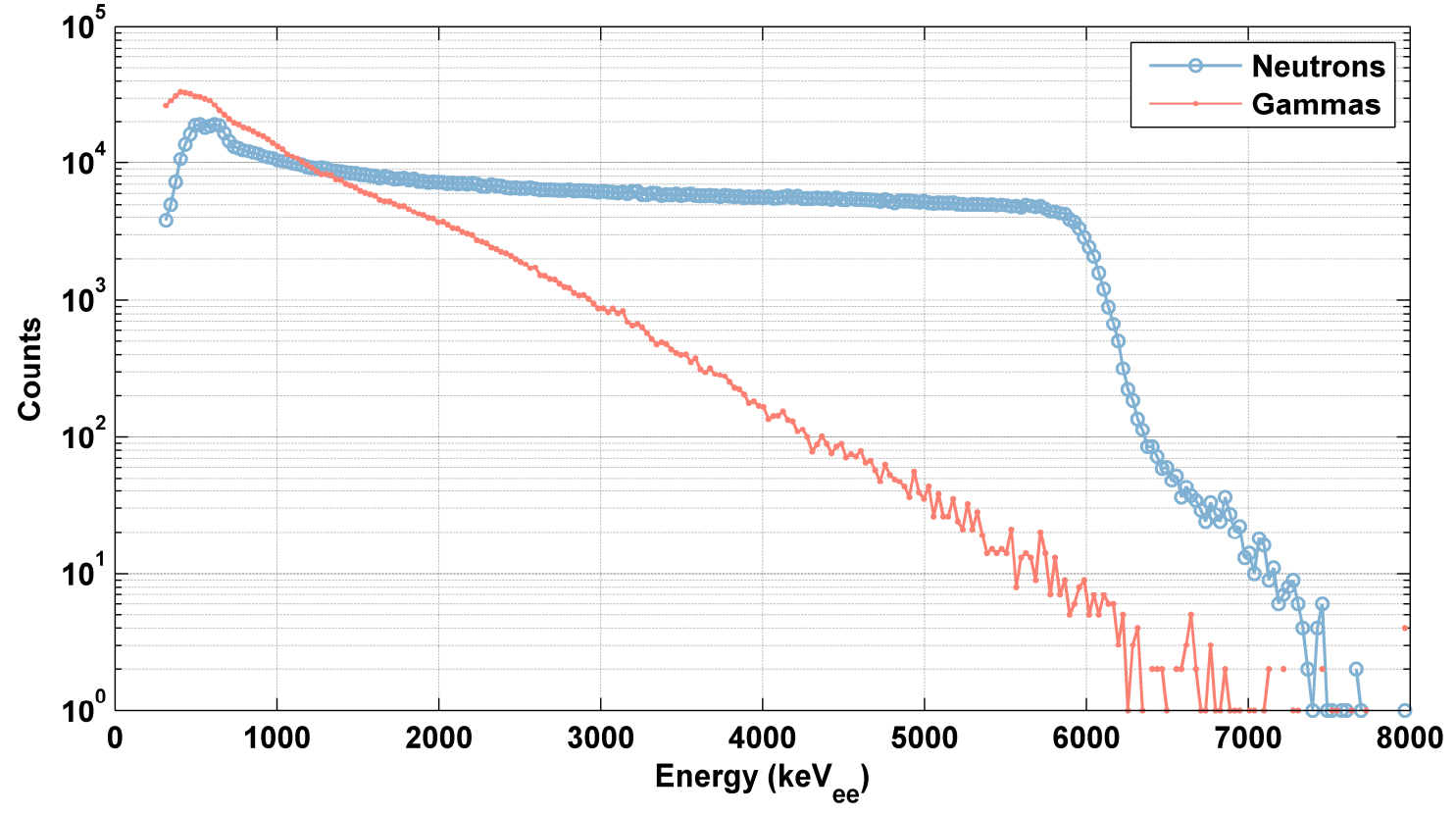

Figure 5: Separated neutron and energy gamma spectrum.

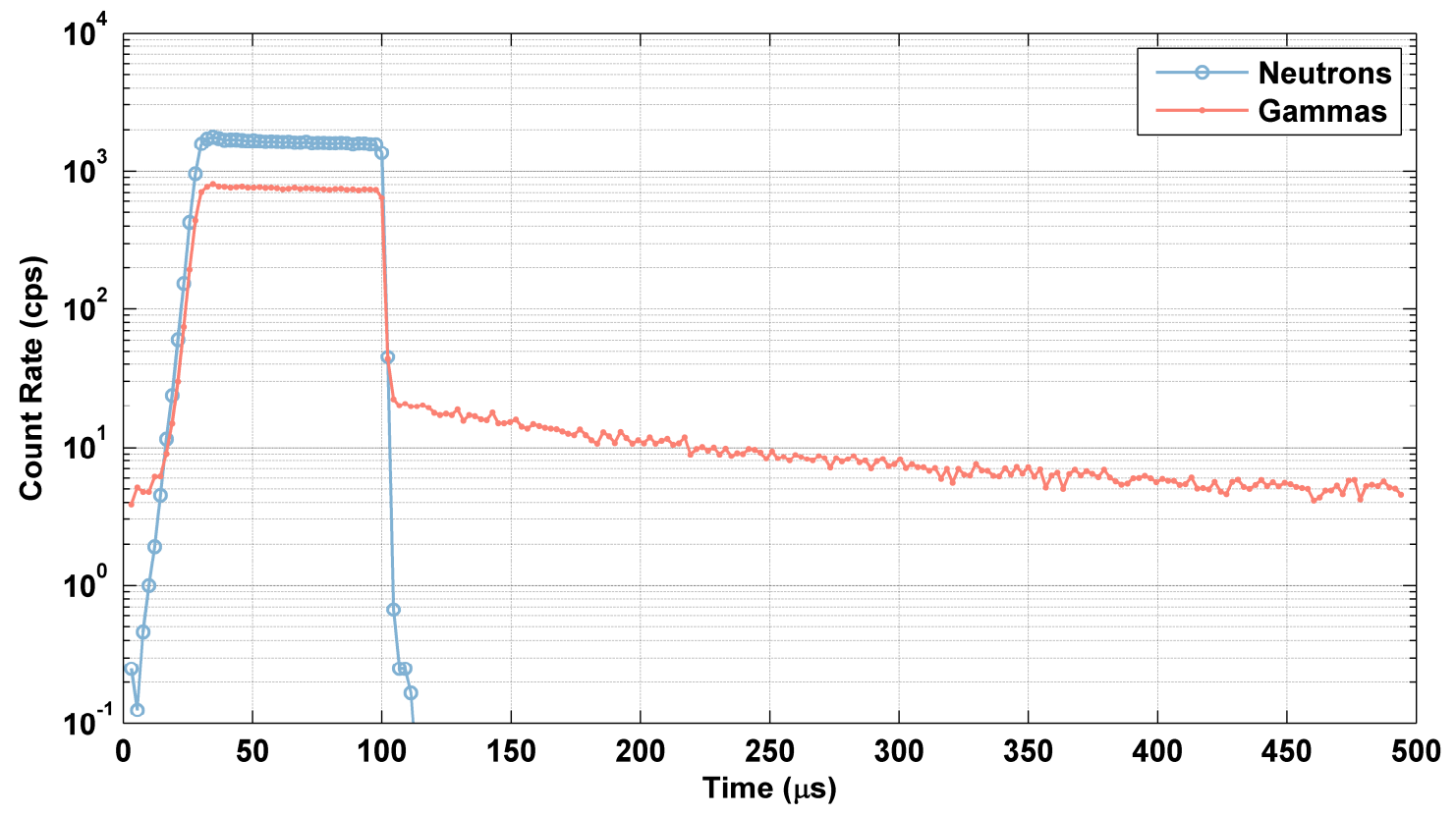

Figure 6: Fast neutron and gamma count rates over the course of a $2 \mathrm{kHz}, 20 \%$ duty cycle burst period. 
The neutron burst profiles measured with a $5 \mathrm{KHz}$ pulse rate at varying duty cycles are overlaid in Figure 7. It is apparent that the neutron generation does not respond immediately to the pulse generator gate. There is a delay prior to the generation of the earliest neutrons, with the output increasing exponentially before approaching equilibrium. The shape of the burst profiles were thought to be due to the switch-on (striking) characteristics of the Penning ion source. After the ionization becomes self-sustaining, the exponential climb of the output $N$ over time $t$ is characterized by the ionization time constant $\tau_{i}[13,38]$ :

$$
N(t)=N_{0} e^{t / \tau_{i}} .
$$

The leading edge of each burst profile was fitted with the above formula to determine the ionization time $\tau_{\mathrm{i}}$. The delay time $\mathrm{t}_{\mathrm{d}}$, measured between the switch on and the time where the neutron output reached $50 \%$ of its equilibrium value was also evaluated. The switch off of the neutron source was comparatively fast and similar for all pulsing schemes.

The timing characteristics for the various pulsing modes are reported in Table 1 . The burst count rate describes the neutron count rate during the neutron burst (once the neutron generation rate has reached equilibrium). Equation (2.3) was used to estimate the true count rates, where the dead times were up to $2 \%$ of the true time within the burst duration. At reduced duty cycles, the generator needs to increase the burst rate to maintain the same average output. As the equilibrium ionisation rate of a Penning ion source is proportional to the gas pressure [39], the generator responds by increasing the gas pressure in the tube. The mean ionisation time $\tau_{i}$ and delay time $t_{d}$ were both inversely proportional to the burst rate, consistent with the model that higher gas pressures allow the ionisation to both initiate and propagate in a shorter time $[13,38]$.

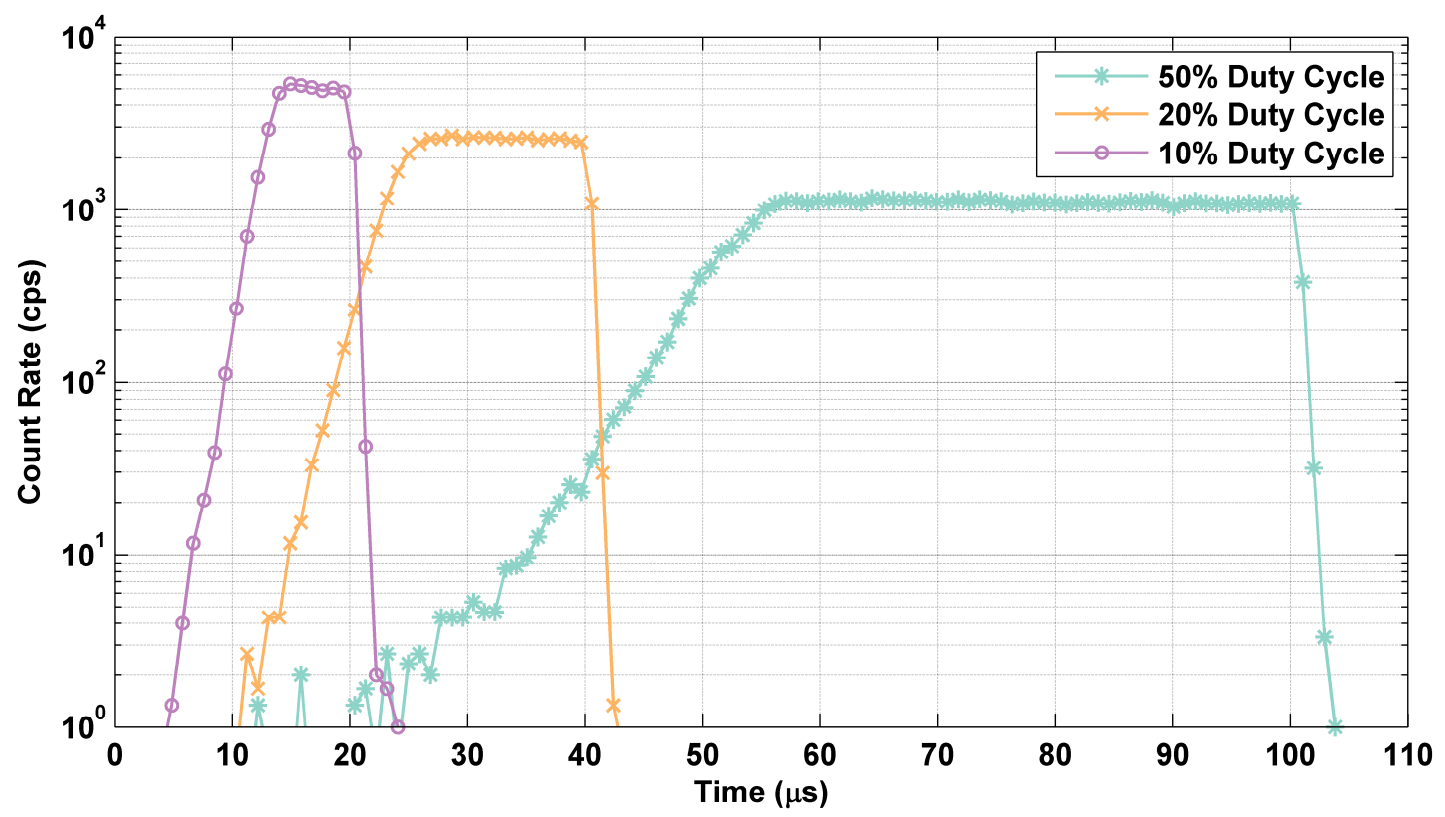

Figure 7: Neutron output profiles at a $5 \mathrm{kHz}$ pulse rate with various duty cycles. 
Table 1: Switching Characteristics of the A-325 STNG Operated in Various Pulsing Schemes.

\begin{tabular}{|c|c|c|c|c|c|}
\hline \multicolumn{2}{|c|}{ Pulsing Scheme } & \multicolumn{2}{|c|}{ Neutron Count Rates } & \multicolumn{2}{|c|}{ Striking Times } \\
\hline $\begin{array}{c}\text { Frequency } \\
\mathbf{k H z}\end{array}$ & $\begin{array}{c}\text { Duty Cycle } \\
\% \\
\end{array}$ & $\begin{array}{c}\text { Average } \\
\text { cps }\end{array}$ & $\begin{array}{c}\text { Burst } \\
\text { cps }\end{array}$ & $\begin{array}{c}\text { Ionisation } \tau_{i} \\
\mu \mathrm{S}\end{array}$ & $\begin{array}{c}\text { Delay } t_{d} \\
\mu \mathrm{S} \\
\end{array}$ \\
\hline 1 & 10 & 233 & 2792 & 1.6 & 18.5 \\
\hline 1 & 20 & 283 & 1623 & 2.3 & 30.2 \\
\hline 1 & 50 & 320 & 733 & 7.0 & 74.0 \\
\hline 2 & 10 & 218 & 3097 & 1.4 & 16.3 \\
\hline 2 & 20 & 267 & 1767 & 2.4 & 27.4 \\
\hline 2 & 50 & 309 & 816 & 6.1 & 67.0 \\
\hline 5 & 10 & 200 & 5178 & 1.1 & 13.1 \\
\hline 5 & 20 & 226 & 2578 & 1.7 & 23.6 \\
\hline 5 & 50 & 278 & 1111 & 4.7 & 52.2 \\
\hline
\end{tabular}

\section{Conclusions}

A prototype neutron detection system, based on an EJ-299-34 plastic scintillator, SiPM optical readout and DSP digital pulse processor, was used to measure the output of a Thermo-Fisher A-325 STNG in various pulsing configurations. The timing capabilities of the digital pulse processer allowed the neutron generation rate over the course of the pulsing cycle to be measured. The pulse shape discrimination property of the plastic scintillator allowed the contributions from fast-neutrons and gammas to be separated using digital pulse shape analysis.

The delay time of the neutron output was found to depend strongly on the pulsing scheme used. The neutron burst delay time ranged from $13.1 \mu$ s to $74.0 \mu$ s for the tested settings. The variable timing of the turn-on process has consequences for how the detection systems in an apparatus relying on neutron pulsing should be set up and optimized. As the effective duty cycle of the neutron output is lower than that of the HV operating the ion source, the dead time of a detector measuring the fast neutrons or associated gammas can be significantly higher than expected. The measured burst timing data will be used in modeling and optimization of prototype apparatus using the A-325 in pulsed mode.

As the demonstrated neutron detector is relatively uncomplicated and inexpensive, it is potentially appropriate for inclusion in the final apparatus. This would allow both monitoring of the mean output of the STNG and characterization for the short-term burst characteristics providing a useful diagnostic in applications where the STNG is used in a range of pulsing and output configurations.

\section{Acknowledgments}

The authors would like to thank Charles Hurlbut from Eljen Technology for kindly providing the EJ-299-34 developmental plastic scintillators used in this work.

\section{References}

[1] A. Buffler, Contraband detection with fast neutrons, Radiat. Phys. Chem 71 (2004) 853-61 
[2] A. Buffler and J. Tickner, Detecting contraband using neutrons: Challenges and future directions, Radiat. Meas. 45 (2010) 1186-92

[3] L. Shope, R. Berg, M. L. O'Neal and B. Barnaby, The operation and life of the Zetatron neutron tube in a borehole logging application, Int. J. Appl. Radiat. Is. 34 (1983) 269-72

[4] D. Ellis and J. Singer, Well logging for earth scientists, Springer 2008

[5] N. G. Cutmore, Y. Liu and J. R. Tickner, Development and commercialization of a fast-neutron/xray Cargo Scanner, in proceedings of IEEE International Conference on Technologies for Homeland Security (2010) 330-6

[6] J. E. Eberhardt, S. Rainey, R. J. Stevens, B. D. Sowerby and J. R. Tickner, Fast neutron radiography scanner for the detection of contraband in air cargo containers, Appl. Rad. Isotopes 63 (2005) 179-88

[7] A. S. Tsybin and A. E. Shikanov, Neutron generation in small sealed accelerating tubes, Sov. Phys. J. $281985609-32$

[8] J. Csikai, Utilization of intense neutron generators in science and technology, Nucl. Instrum. Methods Phys. Res. A 280 (1989) 233-50

[9] G. Vourvopoulos and P. C. Womble, Pulsed fast/thermal neutron analysis: a technique for explosives detection, Talanta 54 (2001) 459-68

[10] L. Allen, W. Mills and D. C. Stromswold, Pulsed Neutron Porosity Logging Using Epithermal Neutron Lifetime, The Log Analyst 30 (1989) 3

[11] D. Hilchie, W. Mills, C. Dennis and W. Givens, Some aspects of pulsed neutron logging, The Log Analyst (1969) 7-17

[12] K. A. Jordan and T. Gozani, Pulsed neutron differential die away analysis for detection of nuclear materials, Nucl. Instrum. Methods Phys. Res. B 261 (2007) 365-8

[13] A. D. Liberman and F. K. Chen, Neutron-burst timing with sealed tube generators, Proc. SPIE 2339 (1995) 188-95

[14] R. Bencardino and J. E. Eberhardt, Development of a fast-neutron detector with silicon photomultiplier readout, IEEE Trans. Nucl. Sci. 56 (2009) 1129-34

[15] E. Burns and G. Bischoff, Ion sources for sealed neutron tubes, AIP Conf. Proc. 392 (1997) 12071210

[16] F. D. Brooks, Development of organic scintillators, Nucl. Instrum. Methods 162 (1979) 477-505

[17] H. Klein and F. Brooks, Scintillation detectors for fast neutrons, in proceedings of International workshop on fast neutron detectors and applications PoS (FNDA2006) 097

[18] N. Zaitseva et al., Plastic scintillators with efficient neutron/gamma pulse shape discrimination, Nucl. Instrum. Methods Phys. Res. A 668 (2012) 88-93

[19] A. Favalli et al., Pulses shape discrimination properties of neutron-sensitive organic scintillators, IEEE Trans. Nucl. Sci. 60 (2013) 1053-6

[20] I. Kreslo et al., Pulse-shape discrimination of scintillation from alpha and beta particles with liquid scintillator and Geiger-mode multipixel avalanche diodes, 2006 JINST 6 P07009-P07009

$$
-11-
$$


[21] R. Preston, J. E. Eberhardt, R. Bencardino and J. Tickner, Software-based digital pulse processing for silicon photomultiplier radiation detectors, in IEEE Nuclear Science Symposium and Medical Imaging Conference Record (2012) pp 462-7

[22] B.S. Budden et al., Analysis of Cs2LiYCl6:Ce3+ (CLYC) waveforms as read out by solid state photomultipliers, in IEEE Nuclear Science Symposium and Medical Imaging Conference Record (2012) pp 347-50

[23] J. Glodo et al., Integrated Neutron Detector for Handheld Systems, IEEE Trans. Nucl. Sci. 60 (2013) 903-7

[24] P. Eckert, H. Schultz-Coulon, W. Shen, R. Stamen and A. Tadday, Characterisation studies of silicon photomultipliers, Nucl. Instrum. Methods Phys. Res. A 620 (2010) 217-226

[25] E. Garutti, Silicon photomultipliers for high energy physics detectors, 2011 JINST 6 C10003

[26] N. Dinu et al., Electro-optical characterization of SiPM: A comparative study, Nucl. Instrum. Methods Phys. Res. A 610 (2009) 423-6

[27] E. Gatti and F. De Martini, A New Linear Method of Discrimination Between Elementary Particles In Scintillation Counters, in Nuclear Electronics II (1962) 265-276

[28] P. A. Söderström, J. Nyberg and R . Wolters, Digital pulse-shape discrimination of fast neutrons and $y$ rays, Nucl. Instrum. Methods Phys. Res. A 594 (2008) 79-89

[29] E. Gatti and F. De Martini, Optimum linear filter for pulse shape recognition in the presence of thermal noise, Energia nucleare 9 (1962) 160-163

[30] J. H. Holland, Adaption in natural and artificial systems, MIT Press, 1992

[31] G.F. Knoll, Radiation detection and measurement, $3^{\text {rd }}$ edition, John Wiley $\&$ Sons Inc., 2000

[32] J. Hunter, Matplotlib: A 2D graphics environment, Comput. Sci. Eng. 9 (2007) 90-95

[33] PySerial, available: http://pyserial.sf.net

[34] Y. Oyama, S. Tanaka and K. Tsuda, A small spherical NE213 scintillation detector for use in inassembly fast neutron spectrum measurements, Nucl. Instrum. Methods Phys. Res. A 256 (1987) $333-8$

[35] S. Nyibule et al., Radioluminescent characteristics of the EJ 299-33 plastic scintillator, Nucl. Instrum. Methods Phys. Res. A 728 (2013) 36-9

[36] D. Smith, R. Polk and T. Miller, Measurement of the response of several organic scintillators to electrons, protons and deuterons, Nucl. Instrum. Methods 64 (1968) 157-66

[37] M. Grodzicka et al., Energy resolution of small scintillation detectors with SiPM light readout, 2013 JINST 8 P02017

[38] F.K. Chen, Investigation of the striking characteristics of a Penning ion source, J. Appl. Phys. 56 (1984) 3191-97

[39] B. F. Gavin, PIG Ion Sources, in The Physics and Technology of Ion Sources, John Wiley \& Sons 1989 